Kiel

\title{
ÜBERLEGUNGEN ZUR ENTSTEHUNG DES BESTIMMTEN ARTIKELS IM ROMANISCHEN
}

\section{SPRACHTHEORETISCHE VORBEMERKUNGEN}

Sprachwandel ist das unabwendbare, ungewollte Ergebnis natürlicher, spontaner Sprech (und Hör)tätigkeit. ${ }^{1}$ Sprechtätigkeit ist ihrerseits geprägt durch

- das individuelle Sosein der Sprecher/Hörer und deren Einbettung in jeweilige gesellschaftlich-historische Lebensumstände;

- das hic et nunc gegebene sprachliche System $\left(\mathrm{L}_{\mathrm{i}}\right.$ oder langue) als strukturiertes Repertoire sprachlicher Ausdrucksmittel und -zwänge;²

- die allen sprachlichen Systemen inhärente Dynamik, die in allgemein menschlichen Maximen sprachlichen Handelns ${ }^{3}$ ihre Ursache hat, dabei aber in jeder $\mathrm{L}_{\mathrm{i}}$ strukturspezifische Ausprägungen erfährt.

Als Sprachwandelphänomen hat die Artikelentstehung im Romanischen einerseits universale Gegebenheiten als notwendige (wenngleich nicht hinreichende) Voraussetzung, und sie weist Parallelen in anderen Sprachfamilien auf, ist also ein Fall unter Fällen - und hat doch auch andererseits als historischer Vorgang ihre unverkennbare Eigenart: sie ist "vergleichbar und unvergleichlich" (M. Wandruszka) je nachdem, ob wir sie sub specie universalitatis oder sub specie individualitatis betrachten.

\section{BIBLIOGRAPHISCHE VORBEMERKUNGEN}

Ebenso janusköpfig wie das Problem ist die dazu vorliegende umfangreiche $\mathrm{Li}$ teratur; diesbezüglich sei hier vor allem auf die Arbeiten einerseits von R. Ultan, ${ }^{4}$ andererseits von Chr. Schmitt, ${ }^{5}$ J. Garrido ${ }^{6}$ und M. Selig ${ }^{7}$ hingewiesen.

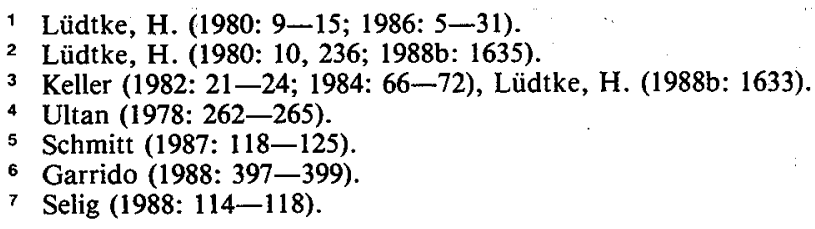




\section{ARTIKELENTSTEHUNG ALS ALLGEMEINER FALL}

Im Rahmen des universalen Sprachwandelgesetzes ${ }^{8}$ mit seinen drei Grundprozessen, ${ }^{9}$ die sich aus Maximen sprachlichen Handelns herleiten (s. 1.),

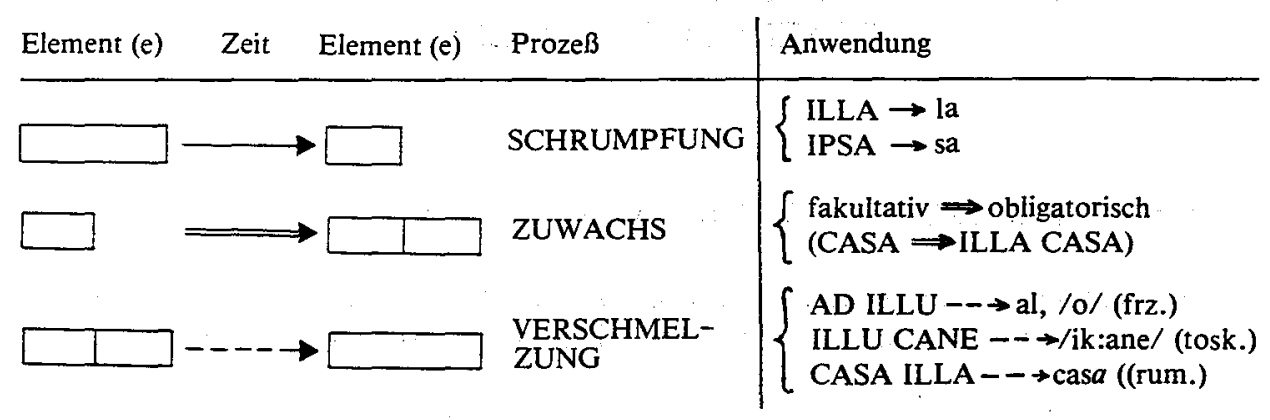

stellt die Artikelentstehung einen prototypischen Fall dar. Ebenfalls liegen die für Grammatikalisierung von Lexemen charakteristischen Phänomene ${ }^{10}$ vor. Der entscheidende Schritt vom "Noch-nicht-Artikel" zum Artikel (oder zum "Artikoloid", s. dazu 7.3.) scheint mir darin zu bestehen, daß die pragmatisch motivierte, d.h. vom Sprecher fallweise erwogene Sețung oder Nichtsetzung eines nominalen Determinanten (z.B. ILLE) übergeht in syntaktische Routine (ILLE : UNUS : $\varnothing$ als Selektionszwang ${ }^{11}$ ). Der Unterschied muß in der Art der Verarbeitung sprachlicher Mittel im Gehirn liegen und ist daher wohl nur intuitiv faßbar, nicht aber in Texten belegbar.

Der Prozeß, der zur Artikelentstehung führt, verläuft irreversibel ${ }^{12}$ und ziellos; der entstandene Artikel schrumpft lautlich immer weiter und kann sogar zu Null werden: im Rumänischen sind negru "schwarz" und negrul "der Schwarze" sowie membri "Mitglieder" und membrii "die Mitglieder" homophon; ähnliches gilt in frankoprovenzalischen Mundarten des Kantons Wallis für vokalisch anlautende Substantive, z.B. èrba "(das) Gras". ${ }^{13}$ Parallel dazu verliert der Artikel mehr und mehr an Informationsgehalt; so zeigt die Endung - $a$ (bzw. $-i$ ), die im klassischen Aramäisch noch die Funktion eines bestimmten Artikels hatte, in modernen aramäischen Mundarten nur noch an, daß das vorangehende Lexem ein Substantiv ist. ${ }^{14}$ Das ist die letzte Etappe vor dem völligen Unkenntlichwerden; J. Greenberg ${ }^{15}$ spricht

8 Lüdtke, H. (1980: 14f.; 1986: 15).

9 Lüdtke, H. (1980: 187-216; 1986: 14-31).

10 Lüdtke, H. (1980: 234-247; 1984: 756-758; 198b: 1634-1638), Lehmann (1982: 21, passim; 1985: 43-45), Werner (1984a: 210-217; 1984b: 541-544).

11 Lüdtke, H. (1979: 73; 1988b: 1635).

12 Lüdtke, H. (1980: 239).

13 Bjerrome (1957: 59).

14 Tsereteli (1970: 37), Maclean (1895/1971: § 16).

15 Greenberg (1978: 59-61). 
denn auch vom "Zyklus" des Artikels. Entstehung und Untergang sind Phasen eines und desselben Prozesses.

\section{DEFINITHEIT UND ARTIKELGEBRAUCH}

Geht man von der naiven Vorstellung aus, es gäbe eine universale Kategorie "Definitheit", die in manchen Sprachen unbezeichnet bleibe, in anderen durch den bestimmten Artikel bezeichnet werde, gerät man rasch in eine Sackgasse, weil die einzelnen Artikelsprachen sich im Gebrauch des Artikels in vielfältiger Weise unterscheiden; ein paar Beispiele mögen diesen Sachverhalt veranschaulichen:

engl. at Øschool

ital. a Øscuola

rum. la şcoaläØ. dt. in der Schule

frz. à l'école

span. en la escuela

frz. l'or est un métal précieux

engl. $\quad$ gold is a precious metal

arab. Ømalik $\varnothing$ yaum $a d$-din

dt. (wörtlich) '(der) König (des) Tag(es) des Glaubens'

"der Herr über das jüngste Gericht"

rum. m-am uitat la carte $\varnothing$

frz. j'ai regardé le livre

dt. er wurde zum Minister ernannt

frz. il fut nommé Ǿministre

engl. he has $Ø$ blue eyes

ital. ha gli occhi azzurri ${ }^{16}$

Es entsteht infolgedessen ein Dilemma: entweder bezeichnet der bestimmte Artikel hier stets die Definitheit und nichts als die Definitheit; dann aber ist Definitheit etwas einzelsprachlich Verschiedenes, so daß wir zu einer Tautologie kommen oder aber wir betrachten Definitheit als universale pragmatische Kategorie und definieren unabhängig davon den (bzw. die) Artikel ${ }^{17}$ als grammatische Kategorie mancher Sprachen, nämlich als minimalen Substantivdeterminanten ${ }^{18}$ mit einzelsprachlich etwas unterschiedlich (sei es syntaktisch, sei es semantisch) geregelter Verwendung. In diesem letzteren Fall gilt es zu klären, was "Definitheit" ist und in welcher Beziehung dazu der bestimmte Artikel steht.

16 Weitere Beispiele gibt Krámský (1972: passim).

17 Harris, M. (1977: 255-260; 1980b: 151-153).

18 Es gibt auch Sprachen, welche zwei verschiedene Sorten von bestimmten Artikeln verwenden je nachdem, woher die Definitheit resultiert; das ist der Fall in niederrheinischen Mundarten, vgl. Heinrichs (1954: 85-103), sowie im Nordfriesischen, vgl. Ebert (1971: 73ff.). 


\section{DEFINITHEIT ALS PRAGMATISCHE KATEGORIE}

Definitheit liegt vor, wenn ein Referent individualisiert und sowohl vom Sprecher als auch (gemäß sprecherseitiger Mutmaßung) vom Hörer identifiziert ist. ${ }^{19}$ Diese Definition trifft zu für Zählbares (z.B. "Haus") einschließlich Unikate (z.B. "Sonne" im nicht-astronomischen, "Gott" im monotheistischen Diskurs), nicht hingegen für Substanzen (z.B. "Gold") sowie für Abstrakta (z.B. "Wissen") und auch nicht für Klassenbezeichnungen (z.B. "der Wolf ist ein Säugetier"). Diese unterschiedlichen kognitiven Voraussetzungen erklären einige - aber nicht alle - von Sprache zu Sprache unterschiedlichen Verwendungen des bestimmten Artikels.

\section{VERWENDUNG UND LEISTUNG DES BESTIMMTEN ARTIKELS}

Indem wir von dem Vorwissen ausgehen, daß mit dem bestimmten Artikel in den meisten Fällen nur bzw. auch Definitheit bezeichnet wird, können wir die Fragestellung einengen und nun untersuchen,

- welche Funktionen der bestimmte Artikel sonst noch hat (a) und

- welche anderen sprachlichen Mittel in Konkurrenz zu ihm ebenfalls die Definitheit bezeichnen (b).

$\mathrm{Zu}$ (a) finden wir die Bezeichnung von:

- Numerus (frz. la croix : les croix; dt. der Löffel : die Löffel)

- Genus (span. el bueno "der Gute" : lo bueno "das Gute")

- Kasus (rum. omul "der Mann" : omului "des Mannes"; arab. kitāb al-malik "das Buch des Königs"; dt. die Frau : der Frau)

- Kongruenz der dem Substantiv nachgestellten attributiven Adjektive (arab. $a l$-gumhūriyya al-'arabiyya al-muttahida "die Vereinigte Arabische Republik“).

$\mathrm{Zu}$ (b) finden wir als Konkurrenten des bestimmten Artikels neben anderen Determinanten (z.B. Possessiva, Demonstrativa: frz. les hommes : tes hommes : ces hommes-là) vor allem Präpositionen; vgl. rum. exploatarea omului de către omø / frz. l'exploitation de l'homme par l'homme; arab. kitāb min kutub al-malik, wörtlich "(ein) Buch von (den) Büchern des Königs" d.h. "ein Buch des Königs“. Im Finnischen können auch Kasusoppositionen die Bezeichnung der Definitheit übernehmen. ${ }^{20}$

Ultan (1978: 249f:), Seiler (1978: 315).

20 Ultan (1978: 254-257). 


\section{ARTIKELENTSTEHUNG IM LATEINISCH-ROMANISCHEN ALS HISTORISCHER PROZEB}

Wann? wie? warum? - So könnte man den Fragenkomplex kurz und bündig zusammenfassen, wenn man klare und einfache Antworten erwartete. Stattdessen müssen wir den Vorgang der Artikelentstehung selbst schon als ein verflochtenes Bündel mehrerer Einzelvorgänge ins Auge fassen, die ihre je eigenen Abläufe (d.h. chronologische Etappen, Motivationen, Ursachen) haben. Wir werden also analytisch verfahren und nacheinander folgende Teilaspekte diachronisch behandeln:

(a) den etymologischen, nämlich das Schicksal von ILLE (bzw. ILLE und IPSE) in attributivem Gebrauch, ${ }^{21}$ wobei wir ein ständiges Anwachsen (Frequenzzunahme) feststellen;

(b) den onomasiologischen, indem wir "Definitheit" als invariante pragmatische Gegebenheit (s. 4-5.) voraussetzen und fragen, ob - und wenn ja, mit welchen Mitteln - sie im lateinisch-romanischen Sprachkontinuum bezeichnet worden ist;

(c) den funktionellen, indem wir Substantivdetermination als morphosyntaktisches (Sub)System in seinen raumzeitlichen Wandlungen betrachten.

\subsection{Die romanischen Fortsetzer von ILLE und IPSE}

Wenn wir mit dem Begriff der diachronischen (etymologischen) Identität operieren, können wir sagen, daß in frz. $d u$ vin das Lexem ILL- steckt, und zwar attributiv gebraucht. Dehnen wir die Betrachtung (im Zeitraffertempo) auf die Gesamtromania aus, können wir zunächst feststellen, daß es in allen romanischen Mundarten den bestimmten Artikel gibt, und sodann drei Ergebniszustände unterscheiden, nämlich aufgrund seiner Entstehung

- aus nachgestelltem ILLE (rumänisch)

- aus vorangestelltem IPSE (sardisch sowie drei Teilgebiete des OkzitanischKatalanischen ${ }^{22}$ )

- aus vorangestelltem ILLE (übrige Romania).

Diese diatopische Variation soll im folgenden vernachlässigt werden, weil sie für das Problem der Artikelentstehung unwichtig ist; die Betrachtung richtet sich ausschließlich auf attributives ILLE ungeachtet seiner Position.

21 In etymologischer Hinsicht ist in den meisten romanischen Sprachen der bestimmte Artikel mit dem Personalpronomen der 3. Pers. identisch, ähnlich wie Demonstrativa zumeist (d.h. mit Ausnahme des Französischen) sowohl attributiv als auch pronominal verwendet werden können, vgl. dazu Harris, M. (1980a: 77; 1980b: 152).

22 Es handelt sich um die Balearen, einen Teil der Costa Brava (Badia 1951: § 136) sowie im Departement Alpes-Maritimes das Gebiet um Grasse, Puget-Téniers und Castellane (Artikelformen sou - sa - sei), vgl. Ronjat (1937 Bd. III: §§ 533-534). 
Im Lauf der lateinisch-romanischen Sprachgeschichte hat ILLE immer mehr Verwendungen erlangt; das soll nachstehend anhand typischer Beispiele schematisch dargestellt werden.

\section{Tabelle 1}

\begin{tabular}{llc} 
Sprache & Satzbeispiel & $\begin{array}{c}\text { Anzahl } \\
\text { ILLE }\end{array}$ \\
\hline Latein & SOL LUMEN DAT & 0 \\
Rumän. & soarele da lumină & 1 \\
Ital. & il sole da luce & 1 \\
Franz. & le soleil donne de la lumière & 2
\end{tabular}

Tabelle 2

Latein LUMEN LUNAE VENIT A SOLE

Rumän. lumina lunii vine de la soare

Ital. la luce della luna viene dal sole $\quad 3$

Franz. $\quad$ la lumière de la lune vient $\mathrm{d} u$ soleil $\quad 3$

Tabelle 3

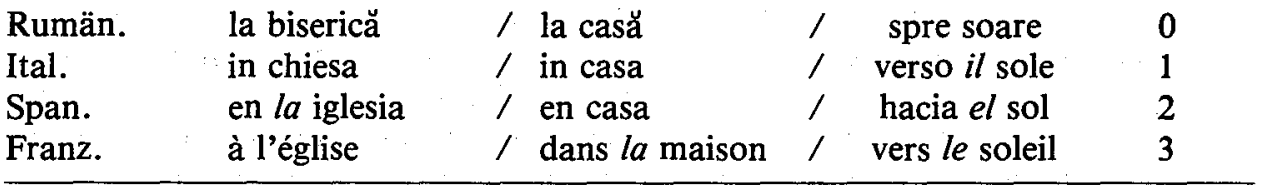

Bei aller grundsätzlichen Ähnlichkeit im Gebrauch des bestimmten Artikels unterscheiden sich die romanischen Sprachen in Einzelheiten, und zwar nicht stochastisch (streuend), sondern quantitativ-systematisch; dabei nehmen Französisch und Rumänisch die beiden Enden der Skala ein, während die übrigen romanischen Sprachen sich (mit geringen Unterschieden voneinander) dazwischen verteilen.

Die Sonderstellung des Französischen im vorliegenden Fall ist ganz offensichtlich Teil einer allgemeinen Charakteristik dieser Sprache, nämlich eines wesentlich rascheren Entwicklungstempos hinsichtlich der drei irreversiblen Grundprozesse des Sprachwandels: Schrumpfung, Anreicherung, Verschmelzung (s. 3.); ${ }^{23}$ damit zusammen hängen grundlegende Strukturwandlungen. ${ }^{24}$

23 Lüdtke, H. (1980: 187-216; 1986: 14-31).

24 Baldinger (1968: passim), Eckert (1986: 354-365; passim). 
Im großen und ganzen läßt sich der romanische Gebrauch des bestimmten Artikels (ähnlich wie der deutsche) als ein sprachlicher Ausdruck für den logischen Allquantor auffassen. ${ }^{25}$ Das Französische hat diese Etappe allerdings schon hinter sich gelassen, wenn es ILLE auch zur Bezeichnung von Teilmengen verwendet ( $d u$ vin; de la lumière; des maisons), und zwar in Verbindung mit der Präposition de ("Partitiv“26). In dieser Konstruktion liegt der eigentliche Ausdruck für die Teilmenge bei der Präposition (vgl. den katalanischen Gebrauch von de + Adjektiv und de + Quantor), während ILLE hier nur noch als obligatorischer Begleiter das nachfolgende Lexem als Substantiv kennzeichnet.

Ein Verfahren der Anreicherung ist neben dem französischen Partitiv (frz. boire de l'eau okz. bèure d'aigo span. beber agua) auch der Ersatz des bestimmten Artikels durch mit ECCE/ECCUM erweitertes ILLE in der Funktion eines anaphorischen Pronomens, siehe

Tabelle 4

Span. $\quad$ la luz del sol y la de la luna

Rumän. lumina soarelui si $a$ lunii

Ital. la luce del sole e quella della luna

Franz. $\quad$ la lumière du soleil et celle de la lune

Wie schon im Fall der anderen drei Tabellen weist das Rumänische auch hier wiederum den geringeren bzw. geringsten Innovationsgrad auf. Es bewahrt offensichtlich eine relativ frühe Etappe der quantitativen Entwicklungsgeschichte von ILLE.

\section{2. "Definitheit" in onomasiologischer Betrachtung}

Definitheit kann explizit bezeichnet werden durch Possessiva (DOMUS MEA), durch Demonstrativa (HAEC DOMUS) oder mittels Ergänzungen (DOMUS UBI X MANET); sie kann vorausgesetzt sein bei Unikaten (SOL), bei Eigennamen (PETRUS) sowie - im Diskurs - aufgrund von Präsuppositionen: so kann REX interpretiert werden als "König, der zum Zeitpunkt $t_{X}$ in $X$ herrscht" und DOMUS als "Haus, wo X wohnt" (indem der Hörer/Leser davon ausgeht, daß das Land X genau einen König hat bzw. das man/ frau in genau einem Haus wohnt). Definitheit zu bezeichnen kann sich auch durch Konventionen erübrigen, und zwar entweder, indem Indefinitheit positiv bezeichnet wird (REX QUIDAM referiert auf irgendeinen vom Leser/Hörer nicht sofort identifizierbaren König) oder infolge ihrer Kop-

Werner (1978: passim).

Ähnlich im Asturianischen: delles perres, frz.des sous "Geld“; vgl. Cano González (1976: 45). 
pelung mit der funktionalen Satzperspektive; vgl. nachstehende tschechische Beispiele $^{27}$ :

/na stole u okna je jablko/ wörtl. auf Tisch bei Fenster ist Apfel, dt. "auf dem Tisch beim Fenster liegt ein Apfel“;

/jablko je na stole u okna/ wörtl. Apfel ist auf Tisch bei Fenster, dt. "der Apfel liegt beim Fenster auf dem Tisch“;

/jablko je na stole u okna/ wörl. Apfel ist auf Tisch bei Fenster, dt. "der Apfel liegt auf dem Tisch beim Fenster“.

Das antike Latein teilt die Freiheit der Stellung der Phrasen im Satz - und damit ihre Verwendbarkeit für die funktionale Satzperspektive - grosso modo mit den heutigen slavischen Sprachen. Im Romanischen hingegen, wo diese Freiheit stark eingeschränkt worden ist, legt ein kompliziertes Regelsystem fest, wann mithilfe des bestimmten bzw. des unbestimmten Artikels Definitheit bzw. Indefinitheit bezeichnet werden muß. Die Entstehung beider Artikel ging offenbar Hand in Hand.

\subsection{Substantivdetermination}

Der Weg zum bestimmten Artikel steht in enger Beziehung zur Veränderung des Systems der Demonstrativa, derjenige zum unbestimmten Artikel mit dem Ersatz von QUIDAM durch (vorangestelltes) UNUS. Von den einsilbigen Demonstrativa schwindet zunächst IS, später auch HIC (von vereinzelten Relikten abgesehen). Zusammen mit der Tilgung anderer kurzer Lexeme, wie MOX, VIX, VIR, URBS usw. ist das ein natürlicher Vorgang im Rahmen der universalen Sprachwandelgesetze.

Gleichzeitig treten längere Neubildungen auf den Plan bzw. werden bestehende immer häufiger verwendet. ${ }^{28} \mathrm{Im}$ vorliegenden Fall sind es Zusammensetzungen vorhandener zweisilbiger Demonstrativa (ISTE, ILLE) mit der Verstärkungspartikel ECCE bzw. ECCUM, deren Gebrauch zunächst wohl mit einer Zeiggebärde gekoppelt war und dann allmählich symbolisch diese ersetzte. Das Ergebnis war ein systematisches Nebeneinander von einfachem und emphatisch verstärktem Demonstrativum.

In diese Neuerung hinein spielen zwei diatopische Differenzierungen zwischen Norden und Süden der Romania. Zwar stimmt ihr Verlauf nicht genau überein und es gibt Überlappungen, im großen und ganzen jedoch setzen Nordfrankreich, Graubünden und Rumänien ECCE fort, gegenüber ECCUM im Süden - und außerdem bewahren jene Gebiete eine Zweieropposition 'nah : fern' (ISTE : ILLE), während

28 Lüdtke, H. (1980: 205-213; 1986: 23-27). 
der Süden der Romania (vor allem Süditalien, Sardinien und die Pyrenäenhalbinsel) IPSE in das deiktische System dergestalt integriert haben, daß eine Dreieropposition in Anlehnung an die drei Personen beim Verb entstand: ISTE "der hier (bei mir)" IPSE "der da (bei dir)" - ILLE "der dort drüben". Zusammen mit der Unterscheidung von einfachem und emphatischem (markiertem) Demonstrativum ergibt das folgendes System:

\begin{tabular}{ll|l|l} 
Latein & $\begin{array}{l}\text { 1. Person } \\
\text { ECCU-ISTE } \\
\text { ISTE }\end{array}$ & $\begin{array}{l}\text { 2. Person } \\
\text { ECCU-IPSE } \\
\text { IPSE }\end{array}$ & $\begin{array}{l}\text { 3. Person } \\
\text { ECCU-ILLE } \\
\text { ILLE }\end{array}$ \\
\hline Altspan. & $\begin{array}{l}\text { aqueste } \\
\text { este }\end{array}$ & $\begin{array}{l}\text { aqueste } \\
\text { ese }\end{array}$ & $\begin{array}{l}\text { aquel } \\
-\end{array}$ \\
\hdashline Südital. $^{29}$ & $\begin{array}{l}\text { kwistu } \\
\text { stu }\end{array}$ & $\begin{array}{l}\text { kwissu } \\
\text { ssu }\end{array}$ & $\begin{array}{l}\text { kwiddu } \\
\text { (ddu) }\end{array}$
\end{tabular}

Entsprechend finden wir (bei Fehlen von IPSE) im Rumänischen ast, acest, cel, acel, im Altfranzösischen ist, cist, icist, cil, icil usw.

Innerhalb dieses Systems der Demonstrativa verschiebt sich im Laufe der Zeit - parallel zur unabwendbaren lautlichen Schrumpfung - das Frequenzverhältnis zugunsten der längeren Formen, die damit allmählich den Status des Normalen, Unmarkierten bekommen, während die Kurzformen eher dann verwendet werden, wenn eigentlich gar kein Zeigen mehr nötig erscheint, die lokaldeiktische Zuordnung (zur ersten, zweiten bzw. dritten Person) jedoch durch die Situation vorgegeben ist (und gewissermaßen nicht ignoriert werden soll). In den süditalienischen Mundarten ist diese Zwischenetappe noch heute recht gut erhalten. Freilich mag es dem Außenstehenden, der an "klare Verhältnisse", d.h. kategoriale Unterscheidung zwischen Demonstrativum und Artikel, gewöhnt ist, schwerfallen, die mit dem Gebrauch des schwachen Demonstrativums ausgedrückten Nuancen zu erfassen.

Eine Parallele zu den süditalienischen Verhältnissen findet sich im Makedonischen ${ }^{30}$ : knigava / knigata / knigana referieren auf "das Buch bei mir / bei dir / dort drüben", mit der Maßgabe freilich, daß die mittlere Form knigata (wie im Bulgarischen, wo die beiden anderen Formen inzwischen verschwunden sind) auch "das Buch schlechthin" (also ohne Spezifierung) bezeichnen kann. Die Phase des alleinigen Artikels (-ot/-ta/-to/-te) ist also bereits vorprogrammiert. In der Romania abgesehen von Süditalien - ist sie (ebenso wie im Bulgarischen) längst erreicht.

Die Konkurrenz "rivalisierender" schwachtoniger Demonstrativa, die sich im Mittelalter im gesamten okzitanisch-katalanischen Raum nachweisen läst, ${ }^{31}$ erklärt auch,

\footnotetext{
Rohlfs (1949 II: § 494), Lüdtke, H. (1979a: 29f.).

Koneski (1967: 228-230).

31 Aebischer (1948: passim), Löfstedt (1961: 268-270), Selig (1988: 96ff., 107f.).
} 
warum neben ILLE gebietsweise IPSE die Oberhand gewinnen und zum alleinigen bestimmten Artikel werden konnte (s. 7.1.).

Mit dem Erreichen dieser letzten Etappe verliert dann die Unterscheidung zwischen starkem und schwachem Demonstrativum ihren Sinn und löst sich auf; die gebietsweise unterschiedlich überlebenden Formen bilden unbeschadet ihrer etymologischen Herkunft ein semantisch einheitliches System: span. este / ese / aquel; kat. aquest / aqueix / aquell u.ä. mit bewahrter Unterscheidung dreier Personen.

\subsection{Semantischer vs. syntaktischer Wandel}

Beim komplexen Phänomen der Entstehung des bestimmten Artikels laufen zwei Vorgänge zusammen, die nicht notwendig in der gleichen Weise aufeinander abgestimmt sein müssen. Es ist zum einen die semantische "Entleerung ", d.h. Verlust der Lokaldeixis, von dem in der am raschesten sich wandelnden romanischen Sprache, dem Französischen, nach ILLE schließlich auch ECCE-ILLE und ECCE-ISTE betroffen worden sind: ce(t) - cette - ces (sowie celui - celle - ceux) haben ihre deiktische Funktion inzwischen an die Partikeln -ci / -là abgegeben. ${ }^{32}$ Zum anderen ist die Entstehung des bestimmten Artikels ein Vorgang, bei dem die in der Deixis implizit enthaltene "Definitheit" bewahrt bleibt und ein Eigengewicht bekommt, das sie vorher nicht hatte. Das heißt zwar nicht, daß das Ex-Deiktikum nun zum alleinigen Ausdruck der Definitheit avanciert, wohl aber, daß eine ganz neue grammatische Kategorie (eben die "Definitheit") entsteht und daß nun die Verwendung irgendeines Substantivs im Diskurs mit der Vorschrift verbunden wird, seinen Referenten auf [ \pm Definitheit] hin festzulegen. Solche Festlegung beinhaltet ein Regelsystem, das wie alle grammatischen Regelsysteme vom Kleinkind gelernt und dessen Anwendung somit zur Routine wird.

\subsection{Die grammatische Kategorie [ \pm Definitheit] im Romanischen}

Alle romanischen Mundarten stimmen darin überein, daß sie [- Definitheit] entweder durch $\varnothing$ oder durch UNUS, [ + Definitheit] entweder durch $\varnothing$ oder durch ILLE (gegebenenfalls IPSE, s. 7.1.) bezeichnen; nur in Einzelheiten unterscheiden sie sich ein wenig. Als Faustregeln können (vom Französischen abgesehen) gelten:

(a) Numerusabhängigkeit: positive Kennzeichnung sowohl für [- definit] als auch für [ + definit] im Singular (sofern nichts anderes bestimmt ist); Nullkennzeichnung für [ $[-$ definit $]$, positive für $[+$ definit $]$ im Plural.

(b) Abhängigkeit von Aktantenfunktionen (Kasusrollen): Satzsubjekt und Adnominal verlangen positive Kennzeichnung: ersteres im Singular fast immer, letzteres für $[+$ definit $]$.

32 Harris, M. (1977: 251-261), Dees (1971: passim). 
(c) Abhängigkeit vom Präpositionengebrauch (von Sprache zu Sprache verschieden; am stärksten im Rumänischen, vgl. die nachstehenden Beispiele:

"ich habe das Buch angeschaut" vs. "... ein ..."

m-am uitat la carte $\varnothing$ vs. ... la $o$ carte

"der Mann liebt die Frau"

barbatul iubeşte pe femeie oder

barbatul iubește femeia.

Auf weitere Einzelheiten soll hier nicht eingegangen werden, weil sie für das Problem der Artikelentstehung irrelevant sind.

\section{MULTIFUNKTIONALITÄT DES ROMANISCHEN ARTIKELS}

Der Fall des Englischen, wo the nur [ + definit] und $a$ nur [ - definit] kennzeichnet $^{33}$ und sonst nichts, stellt die Ausnahme und nicht die Regel dar. Von den Nebenfunktionen, die der bestimmte Artikel ähnlich wie im Deutschen auch in verschiedenen romanischen Sprachen hat, kann die der Numerusbezeichnung als relativ spät aufgekommener Sonderfall des Französischen (la/les croix), Provenzalischen (la/li crous) und eines Teils des Süditalienischen $(a / i k r u c ́ \text { ) })^{34}$ angesehen werden. Was die Genusbezeichnung betrifft, müssen drei Phänomene unterschieden werden, nämlich (a) lexikalische Homophonie als Zufall (vgl. dt. der/die Leiter): frz. le mur / la mûre, (b) Kennzeichnung des natürlichen Geschlechts bei Referenz auf Personen: frz. le / la concierge und (c) grammatische Kategorie [ \pm zählbar] im AsturischLeonesischen sowie in einem großen Teil von Mittel- und Süditalien ${ }^{35}$, die aus der Opposition ILLUM BONUM : ILLUD BONUM dort entstand, wo lat. Ŭ zu /o/geöffnet wurde ${ }^{36}$. Hier wurde die nur beim entstehenden Artikel lautgesetzlich resultierende formale Unterscheidung $(l u: l o)$ auf Adjektiv und Substantiv übertragen: l $u$ bon $u$ ferr $u$ vs. lo bono ferro.

Ebenso wichtig ist die Rolle des bestimmten Artikels bei der Kasuskennzeichnung. Es ist vielleicht kein Zufall, daß in all denjenigen Gebieten der Romania, wo wir erhaltene Kasus-Oppositionen vorfinden, diese teils nur, teils auch mithilfe des Artikels zum Ausdruck gebracht werden. Man vergleiche nachstehende Übersicht:

Sroka (1981: 196-200).

Rohlfs (1949 1: $\S \S 141,144,147)$, Lüdtke, H. (1979a: 25f.; passim).

35 Lüdtke, H. (1988: 65-69; 1979: 66-68), Rohlfs (1949 II: § 419).

36 Lüdtke, H. (1965: passim; 1988a: 65-69). 


\begin{tabular}{|c|c|c|}
\hline Rumän. & fratele & "der/den Bruder" \\
\hline & fratelui & "des Bruders, dem Bruder" \\
\hline Bündnerrom..$^{37}$ & $i(g) l$ frar & "der/den Bruder" \\
\hline & li/agli frar & "dem Bruder" \\
\hline Altfrz. & li frere(s) & "der Bruder" \\
\hline & lo frere & "dem/den Bruder, des Bruders" \\
\hline Frankoprov. & $l$ frare & "der Bruder". \\
\hline (Wallis) $^{38}$ & $o$ frare & "den Bruder" \\
\hline & $\iota$ šwaira & “die Schwester (Nom.)“ \\
\hline & $a$ šwaira & “die Schwester (Akk.)“ \\
\hline
\end{tabular}

Die Form $l$ des Nom.Sg.Fem. ist vom Maskulinum her übertragen worden, so daß die Opposition nun (außer bei vokalisch anlautenden Substantiven) für den gesamten Singular gilt.

\section{DAS URSPRUNGSPROBLEM - WISSENSCHAFTSTHEORETISCH BETRACHTET}

Seit den Arbeiten von Wolterstorf $\mathrm{f}^{39}$ hat es an Versuchen nicht gefehlt, aus der Untersuchung spätlateinischer Texte chronologisch aufgeschlüsselt den Hergang der Artikelentstehung herauszudestillieren. Aber "die lateinischen Texte reflektieren nur die Anfänge der Artikelentwicklung ... Die spätlateinische Schriftsprache bleibt nach der einmal übernommenen Innovation wiederum hinter der gesprochenen Sprache zurück und perpetuiert die Anfänge der Artikelentwicklung “40.

Das ist im Grunde nicht verwunderlich. Man könnte zwar fragen, ob nicht ähnlich wie in zahlreichen Inschriften die Verfasser unfreiwillig hier und da Interferenzen aus der Spontansprache nachgegeben haben. Das ist jedoch offenbar nicht der Fall. Man muß sich die entsprechende Situation vergegenwärtigen. Der muttersprachliche Sprecher einer Artikelsprache (wir setzen einmal voraus, daß dieser Zustand irgendwann vor dem 8 . Jahrhundert bereits erreicht war) hat - wie die Erlernung z.B. slavischer Sprachen durch Westeuropäer zeigt - keine Mühe, eine artikellose Sprache zu handhaben; jedenfalls ist die Artikellosigkeit als solche kein Problem, denn man braucht nur eine simple Tilgungsregel zu befolgen, wenn man von der Muttersprache ausgeht. Daß im umgekehrten Fall der richtige Gebrauch bzw. Nichtgebrauch des Artikels große Lernschwierigkeiten mit sich bringt, ist für das vorliegende Problem ohne Belang.

Es dürfte also von den ersten nachchristlichen Jahrhunderten an zum lateinischen Elementarunterricht gehört haben, daß man beim Schreiben (wie auch bei der

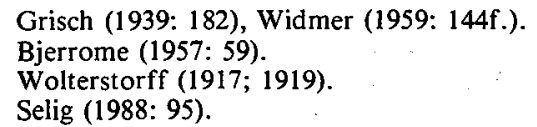


feierlichen Rede) sich die übermäßige Verwendung von Substantivdeterminanten abgewöhnte. Statistik hin - Statistik her: die Widerspiegelung der Artikelentstehung in spätlateinischen Texten finden zu können, bleibt eine utopische Erwartung ${ }^{41}$. Dennoch sollte man nicht ausschließen, daß aufgrund von textlinguistischen und textsortenspezifischen Untersuchungen ${ }^{42}$ doch noch neue Erkenntnisse - und zwar indirekter Art - über dàs Phänomen der Artikelentstehung gewonnen werden.

\section{MOTIVATIONEN UND URSACHEN}

Von der Idee der linearen Monokausalität, d.h. auf unser Problem gewendet: von der Vorstellung, ein und nur ein Umstand könnte die Entwicklung zum Artikel hin in Gang gesetzt haben, müssen wir Abschied nehmen ${ }^{43}$. Sowohl das universale Sprachwandelgesetz der fortwährenden lautlichen Schrumpfung und semantaktischen Anreicherung als auch diskurspragmatische Neuerungen, sowohl die zunehmende Häufigkeit der Phrasenstellung SVO als auch die verstärkt wahrgenommene Möglichkeit, bei Indeklinabilien mittels ILLE ihre Aktantenfunktion (Kasusrolle) im Satz kenntlich zu machen ${ }^{44}$ oder Zählbares von Nichtzählbarem zu unterscheiden - alle diese Gegebenheiten können (müssen aber nicht!) zusammengewirkt haben, um den Wandel von der motivierten Setzung von Determinanten zur syntaktischen Programmroutine des Artikelgebrauchs zu bewerkstelligen. Dabei muß stets unterstellt werden, daß in artikellosen Sprachen schwachtonige Demonstrativa immer schon bereitstehen, um erforderlichenfalls gar nicht lokaldeiktisch, sondern zur Bezeichnung von [ + Definitheit] verwendet zu werden ${ }^{45}$. In dieser Situation konnten schließlich auch Kontaktsprachen, namentlich das Griechische, auf den mündlichen Sprachgebrauch einwirken ${ }^{46}$; dies freilich nicht in Gestalt direkter Übernahme der fertigen grammatischen Kategorie, sondern in Form von (weiterer) Frequenzsteigerung des Determinantengebrauchs.

Ceterum censeo: da die Entstehung neuer grammatischer Kategorien von Sprechern/Hörern durch ihre Kommunikationstätigkeit zwar bewirkt, jedoch nicht geplant wird, also Ergebnis von Prozessen der "unsichtbaren Hand"47 ist, sollte die

41 Die Ansicht der Muller-Schule, wonach spätlateinische Texte die volkssprachliche Entwicklung direkt widerspiegeln (Trager 1932: 5), kann man heute nicht mehr ernst nehmen. Wohl aber läßt sich bei umsichtiger Interpretation (vgl. Löfstedt 1961: 254ff. sowie die Arbeiten von Raible und Selig, s. Anm., 42) indirekt einiger Aufschluß über den Wandel der Volkssprache gewinnen.

42 Raible (1985: 44ff.), Selig (1988: 87ff.).

43 Selig (1988: 109-113).

44 Zahlreiche Beispiele bei Wolterstorff (1917: 208-225; 1919: 64-89), Bouvier (1972: 76-79), Schmitt (1987: 104-109).

45 Krámský (1972: 62) gibt als tschechisches Beispiel dej mi tu knihu! "gib mir das Buch!“; K. Sroka hat mir mündlich ähnliche Beispiele für das Polnische mitgeteilt. Krámský führt (1972: 188-190) weiterhin aus, daß sowohl im gesprochenen Tschechisch als auch im Sorbischen ten - ta - to häufig als Nachahmung des deutschen Artikels verwendet werden; ähnliches wird über die polnischen Dialekte in Oberschlesien berichtet. Für das Serbokroatische vgl. Reichenkron (1966: 342-347).

46 Fehling (1980: 358-363), Schmitt (1987: 116f.).

47 Keller (1982: 7-14; 1984: 67). 
Forschung nach dem Verlauf der Artikelentstehung von vornherein darauf verzichten, nach der Ursache, d.h. nach einer isolierbaren Einzelursache zu fahnden, die sich nicht nur ihrer Feststellung entzieht, sondern die wohl gar nicht vorliegen kann. Was weder ganz zufällig entstanden ist noch geplant war, kann mit aristotelischen Kategorien nicht beschrieben werden. Solche Überlegungen, die auch auf der evolutionären Erkenntnistheorie ${ }^{48}$ fußen, sollten die überkommenen Denkschemata ablösen.

\section{BIBLIOGRAPHIE}

Aebischer, P. 1948. Contribution à la protohistoire des articles ILLE et IPSE dans les langues romanes. In: Cultura Neolatina 8. 181-203.

Badia Margarit, Antonio. 1951. Gramática Histórica Catalana. Barcelona: Noguer S.A.

Baldinger, Kurt. 1968. Post- und Prädeterminierung im Französischen. In: Festschrift Walther von Wartburg zum 80. Geburtstag. Tübingen: Niemeyer. 87$-106$.

Bjerrome, Gunnar. 1957. Le patois de bagnes (Valais). Stockholm: Almqvist \& Wiksell.

Bouvier, E. 1972. Le démonstratif latin ILLE et la formation de l'article défini des langues romanes. In: Cahiers de Lexicologie 21. 75-86.

Cano González, Ana Maria et al. 1976. Gramática Bable. Madrid.

Dees, Anthonij. 1971. Etude sur l'évolution des démonstratifs en ancien et en moyen français. Groningen: Wolters-Noordhoff.

Eckert, Gabriele. 1986. Sprachtypus und Geschichte. Untersuchungen zum typologischen Wandel des Französischen. Tübingen: Narr.

Ebert, Karen Heide. 1971. Referenz, Sprechsituation. Die bestimmten Artikel in einem nordfriesischen Dialekt (Fering). Nordfriisk Instituut. Bräist/Bredstedt.

Fehling, Detlef. 1980. The Origins of European Syntax. In: Folia Linguistica Historica I/2. 353-387.

Garrido, Joaquín. 1988. Sobre la evolución hasta el artículo actual en español. In: Actas del I Congreso internacional de historia de la lengua española (Cáceres 1987). Vol. I. Madrid: Arco. 385-399.

Greenberg, Joseph H. 1978. How does a language acquire gender markers? In: Universals of Human Language. Hrsg. J. Greenberg, Ch. Ferguson, E. Moravcsik. Stanford University Press.

Grisch, Mena. 1939. Die Mundart von Surmeir (Ober- und Unterhalbstein). Beitrag zur Kenntnis einer rätoromanischen Sprachlandschaft. Romanica Helvetica. Vol. 12. Paris: Droz. Zürich-Leipzig: Niehans.

Harris, Martin B. 1977. 'Demonstratives', 'articles' and 'third person pronouns' in French: changes in progress. Zeitschrift für Romanische Philologie 93. 249-261.

48 vgl. Wuketits (1984: 64f. und 70-75) sowie die dort angeführte Literatur. 
Harris, Martin B. 1980a. The marking of definiteness: a diachronic perspective. In: Papers from the 4th International Conference on Historical Linguistics, Hrsg. E. Closs Traugott/F. Labrum/S. Shepherd. Amsterdam: Benjamin. 75-86.

Harris, Martin B. 1980b. The marking of definiteness in Romance. In: Historical Morphology. Hrsg. J. Fisiak. The Hague: Mouton. 142-156.

Heinrichs, Heinrich M. 1954. Studien zum bestimmten Artikel in den germanischen Sprachen. Beiträge zur deutschen Philologie. Bd. 1. Giessen: Schmitz.

Keller, Rudi. 1982. Zur Theorie sprachlichen Wandels. Zeitschrift für germanistische Linguistik 10/. 1-27.

Keller, Rudi. 1984. Bemerkungen zur Theorie des sprachlichen Wandels. Zeitschrift für germanistische Linguistik 12/1. 63-81.

Koneski, Blaze. 1967. Gramatika na makedonskiot literaturen jazik. Skopje.

Krámský Jirí. 1972. The article and the concept of definiteness in language. The Hague/Paris: Mouton.

Lehmann, Christian. 1982. Thoughts on grammaticalization. A programmatic sketch. Vol. I. In: akup. Arbeiten des Kölner Universalien-Projekts Nr. 48.

Lehmann, Christian. 1985. The role of grammaticalization in linguistic typology. In: Language invariants and mental operations. International Interdisciplinary Conference held at Gummersbach/Cologne, Sept. 18-23, 1983. Hrsg. H. Seiler/G. Brettschneider. Tübingen: Narr. 41-52.

Löfstedt, Bengt. 1961. Studien über die Sprache der langobardischen Gesetze. Beiträge zur frühmittelalterlichen Latinität. Stockholm: Almqvist \& Wiksell.

Lüdtke, Helmut. 1965. Die lateinischen Endungen -UM/ -UNT und ihre romanischen Ergebnisse. In: Omagiu lui A. Rosetti. Bukarest. 487-499.

Lüdtke, Helmut. 1979a. Profilo dei dialetti italiani. Vol. 17. Pisa: Pacini.

Lüdtke, Helmut. 1979b. Selektionsgrammatik (am Beispiel des Französischen). In: Bildung und Ausbildung in der Romania Bd. II: Sprachwissenschaft und Landeskunde. Hrsg. R. Kloepfer. München: Fink. 71-84.

Lüdtke, Helmut. 1980. Kommunikationstheoretische Grundlagen des Sprachwandels. Hrsg. H. Lüdtke. Berlin: de Gruyter.

Lüdtke, Helmut. 1984. Ansätze zu einer Theorie des Sprachwandels auf syntaktisch-morphologischer Ebene. In: Sprachgeschichte. Ein Handbuch zur Geschichte der deutschen Sprache und ihrer Erforschung. Hrsg. W. Besch/O. Reichmann/ St. Sonderegger. 1. Halbband. Nr. 54, Berlin: de Gruyter. 753-761.

Lüdtke, Helmut. 1986. Esquisse d'une théorie du changement langagier. In: La Linguistique $22 / 1.3-46$.

Lüdtke, Helmut. 1988a. Metafonía y neutro de materia. In: Actas del I Congreso Internacional de Historia de la Lengua Española, Cáceres 30. 3.-4. 4. 1987. Hrsg. M. Ariza/A. Salvador/A. Viudas. Arcos Libros. 61-69.

Lüdtke, Helmut. 1988b. Grammatischer Wandel. In: Sociolinguistics/Soziolinguistik. An International Handbook of the Science of Language and Society. Hrsg. U. Ammon/N. Dittmar/ K.J. Mattheier. 2. Halbband, Nr. 176, Berlin: de Gruyter. 1632-1642.

Maclean, Arthur J. 1895. Grammar of the dialects of Vernacular Syriac. Cambridge. (reprint Amsterdam 1971). 
Meyerstein, Zlata P. 1972. Czech deictics: pronouns and articles? In: Linguistics 91. 17-30.

Raible, Wolfgang. 1985. Nominale Spezifikatoren ("Artikel") in der Tradition lateinischer Juristen oder: Vom Nutzen einer ganzheitlichen Textbetrachtung für die Sprachgeschichte. In: Romanistisches Jahrbuch 36. 44-67.

Reichenkron, Günter, 1966. Anfänge einer Artikelausbildung im Serbokroatischen? In: Die Welt der Slaven. Vierteljahrsschrift für Slavistil 11. 337-352.

Rohlfs, Gerhard. 1949. Historische Grammatik der italienischen Sprache und ihrer Mundarten. 3 Bände. Bern: Francke.

Ronjat, Jules. 1937. Grammaire Istorique des Parlers Provençaux Modernes. Bd. III. Montpellier: Société des langues Romanes.

Schmitt, Christian. 1987. Die Ausbildung des Artikels in der Romania. In: Latein und Romanisch. Romanistisches Kolloquium I. Hrsg. W. Dahmen/G. Holtus/ J. Kramer/M. Metzeltin. Tübingen: Narr. 94-125.

Seiler, Hansjakob. 1978. Determination: A functional dimension for interlanguage comparison. In: Language Universals. Hrsg. H. Seiler. Tübingen: Narr. 301$-328$.

Selig, Maria. 1988. Die Entwicklung des Determinantensystems im Spätlateinischen. In: Romanistik, Sprachtypologie und Universalienforschung. Hrsg. W. Raible. Tübingen. 87-118.

Sroka, Kazimierz A. 1981. The grammatical category of definiteness. In: Sprache: Formen und Strukturen. Akten des 15. Linguistischen Kolloquiums. Münster 1980. Hrsg. M. Kohrt/J. Lenerz. Tübingen: Niemeyer. 193-202.

Trager, George L. 1932. The use of the Latin demonstratives (especially ILLE and IPSE) up to 600 A.D., as the source of the Romance article. New York: Publications of the Institute of French Studies Inc.

Tsereteli, Konstantin G. 1970. Grammatica di assiro moderno. Neapel.

Ultan, Russel. 1978. On the development of a definite article. In: Language Universals. Papers from the Conference held at Gummersbach/Cologne Oct. 3-8, 1976. Hrsg. H. Seiler. Tübingen: Narr. 249-265.

Werner, Otmar. 1978. Der bestimmte Artikel als All-Quantor. In: Sprache in Gegenwart und Geschichte. Festschrift für Heinrich Matthias Heinrichs zum 65. Geburtstag. Hrsg. D. Hartmann/J. Linke/ O. Ludwig. Köln/Wien: Böhlau. 215-235.

Werner, Otmar. 1984a. Morphologische Entwicklungen in den germanischen Sprachen. Das Germanische und die Rekonstruktion der Indogermanischen Grundsprache. Hrsg. J. Untermann/B. Brogyanyi. Current Issues in Linguistic Theory. Bd. 22. Amsterdam: Benjamin. 181-226.

Werner, Otmar. 1984b. Prinzipien und Methoden historischer Morphologie. In: Sprachgeschichte. Ein Handbuch zur Geschichte der deutschen Sprache. Hrsg. W. Besch/O. Reichmann/St. Sonderegger. 1. Halbband. 535-545.

Widmer, P. Ambros. 1959. Das Personalpronomen im Bündnerromanischen in phonetischer und morphologischer Schau. Bern: Francke.

Wolterstorff, G. 1917. Artikelbedeutung von ILLE bei Apuleius. In: Glotta 8. $197-226$. 
Wolterstorff, G. 1919. Entwicklung von ILLE zum bestimmten Artikel. In: Glotta 10. 62-93.

Wuketits, Franz M. 1984. Evolution, Erkenntnis, Ethik. Folgerungen aus der modernen Biologie. Darmstadt: Wissenschaftliche Buchgesellschaft.

\section{Povzetek}

\section{O NASTANKU DOLOČNEGA ČLENA V ROMANSKIH JEZIKIH}

Nastanek romanskega člena je $v$ svojih podrobnostih enkraten dogodek iz zgodovine neke jezikovne skupnosti, ki se razkraja in postaja jezikovna družina. Istočasno pa gre za nepovrnljiv zgodovinski razvoj, ki ne teče povsod enako, vendar pa po splošnih zakonitostih. Razlike v rabi člena med posameznimi romanskimi jeziki (pri tem najbolj odstopata francoščina in romunščina) se dajo vsaj deloma razumeti kot različne etape za celo Romanijo paralelno potekajočega procesa, namreč neprestano naraščajoče rabe lat. ILLE (oziroma IPSE). Tako zgodovina kakor tudi funkcionalnost romanskega člena se dasta bolje dojeti kot doslej, če pritegnemo k primerjavi slovanske jezike, od katerih eni imajo neki člen, drugi pa ne. 